\title{
Policy Intelligence in the Era of Social Computing: Towards a Cross-Policy Decision Support System
}

\author{
Riccardo Boero ${ }^{1}$, Enrico Ferro ${ }^{2}$, Michele Osella ${ }^{2}$, \\ Yannis Charalabidis ${ }^{3}$, and Euripidis Loukis ${ }^{3}$ \\ ${ }^{1}$ Regione Piemonte, Corso Regina Margherita, 174 - 10152 Torino, Italy \\ ricboero@gmail.com \\ ${ }^{2}$ Istituto Superiore Mario Boella, Via Boggio, 61 - 10138 Torino, Italy \\ \{enrico.ferro, michele.osella\}@ismb.it \\ ${ }^{3}$ University of the Aegean, Gorgyras Str. -83200 Karlovassi, Greece \\ \{yannisx, eloukis\} @aegean.gr
}

\begin{abstract}
The paper presents a policy analysis framework developed through a process of interdisciplinary integration as well as through a process of end users needs elicitation. The proposed framework constitutes the theoretical foundation for the Decision Support Component of a technological platform bringing together Social Media and System Dynamics simulation developed within the PADGETS project. The main novelties introduced have to do with the possibility to provide decision makers with a set of concise, fresh and relevant data in a cost effective and easily understandable way.
\end{abstract}

Keywords: ICT Governance, Policy Intelligence, Policy Modeling, eParticipation, Social Media, Decision Support Systems, Public Sector Innovation, eGovernment, PADGETS.

\section{Introduction}

In the second decade of the millennium European Governments are confronted with three important long-term trends.

1. The combined effect of an increase in the rate of change and in the level of interdependence and interconnectedness among regions, activities and groups is leading to a fast-evolving and unpredictable world characterized by significant levels of complexity and uncertainty. The concept of "liquid modernity" [1] proposed by Zygmunt Bauman represents a useful attempt to frame part of such phenomenon. According to the Polish sociologist, in fact, social forms and institutions no longer have enough time to solidify and cannot serve as frames of reference for human actions and long-term life plans to the extent they served in the past, so individuals have to find other complementary ways to organize their lives.

2. A push towards a more participatory and inclusive style of policy making poses significant challenges in terms of striking the right balance between openness and 
control, defining new and appropriate styles of management and, finally, integrating participatory activities into existing decision making processes.

3. A forecast, for years to come, of low economic growth and financial instability is leading to tighter budget constraints and less room for mistakes in the allocation of tax payers' money for Government's action.

The concurrence of such socioeconomic, institutional and financial trends calls for a reconceptualization of Government's roles and modi operandi.

The creation of the PADGETS (its full title being "Policy Gadgets Mashing Underlying Group Knowledge in Web 2.0 Media") project [2] may be placed in such landscape. The project has been financed in the context of the "ICT for Governance and Policy Modelling" call of the seventh European Framework Program of research (FP7). The main underlying idea of such research endeavor is to bring together social computing with System Dynamics simulation in order to help Governments to render policy making processes more participative and, at the same time, to provide advanced and more effective types of support to public sector decision making processes. In particular, the platform developed within the project blueprint will allow Public Administrations to set up a cost effective participatory process by moving the political discussion from official websites to Social Networks where citizens are already debating. The platform will enable a centralized posting of contents and micro-applications (termed "Policy Gadgets" or, coining a portmanteau, "Padgets") to many different Web 2.0 Social Media at the same time, followed by the collection of various types of users' interactions (e.g., views, likes/dislikes, ratings, comments) and by advanced processing and analysis of resulting data in order to provide effective policy intelligence services based on fresh and relevant information.

The aim of this paper is to present a policy analysis framework for this purpose, developed drawing from theories and concepts belonging to different scientific disciplines. The proposed framework constitutes the theoretical foundation on which the Decision Support Component (DSC) of the PADGETS platform has been designed. The development of such a Decision Support System represents a first attempt to provide policy makers with a set of tools that may be precious in tackling grand challenges discussed at the beginning of this section.

Concluding these introductory comments, the paper is structured in five sections. Section two provides a multidisciplinary theoretical background to the presented work. Section three illustrates pivotal principles underlying the proposed policy analysis framework. Section four explains how such framework has been transformed into a working software tool to be integrated into the PADGETS platform. Finally, section five provides some conclusive remarks on the value proposition of the Decision Support System proposed as well as some directions for stimulating future research.

\section{Theoretical Background about Participative Decision Support Systems in the Public Sector}

All different kinds of organizations, business, public, and non-governmental alike, are becoming aware of soaring complexity in decision making situations [3] [4]. Such 
complexity, inextricably related to the intricacy of systems [5], can be addressed through decision support tools which can enhance the quality of the decision process ${ }^{1}$.

However, in a bevy of situations, multidisciplinary teams, top-notch skilled resources and world class computer suites do not suffice to cope with actual problems: a further need concerns the sharing and "externalization" [6] of tacit knowledge already existing in the society. In fact, collective intelligence emerges as a key ingredient of a "distributed problem-solving" system [7] whose output can significantly enrich the decision process traditionally carried out by experts: in accordance with this trend, politics is progressively moving towards higher public engagement and cooperation in decision making processes.

Even though, as underlined by [8], the number of solutions in the area regarding eDemocracy, e-Participation and related fields is increasing, support systems for decision making are, however, still used mainly in narrow professional circles and have not found their way to political decision makers or to the public. The challenge of successful implementation of DSSs in the public sector, with engagement over the whole spectrum of decision making, is still unmet. In particular, in order to enhance the quality and effectiveness of the decision through knowledge harvesting, simulation of future scenarios and structured comparison of alternatives, DSSs depend on the availability and accessibility of timely, relevant and accurate information [9], which frequently represents the scarce resource.

Since such information may derive from "social sources", new mechanisms are required to enable a public decision process open, transparent and participative in which citizens' contribution is a paramount ingredient characterized by a significant impact. Along these lines, since Web 2.0 applications are already being used in Government not only for soft issues (e.g., public relations, public service announcements) but also for core internal tasks (e.g., intelligence services, reviewing patents, support decision making) [10], it is highly desirable to proceed towards a systematic exploitation of the emerging Social Media by Government organizations in the processes of public policies formulation, aiming to enhance a frictionless eParticipation: by doing this, Governments make a step towards citizens rather than expecting the citizenry to move their content production activity onto the "official" spaces created for e-Participation [11].

To sum up, the implementation of successful e-Governance programs, which heavily leverage on the participative dimension, cannot ignore the presence of DSSs, as computer-based systems that help decision makers confront ill-structured problems through direct interaction with data and analytical models, notwithstanding the access to privileged channels aimed to enable a fully-fledged engagement: the evidence that "it has become impossible to restrict knowledge and its movement to castes of specialists" [12] makes researchers aware that "crowd wisdom" is not merely a Web 2.0 catchy buzzword, but is instead a strategic model to attract an interested and motivated platoon of stakeholders.

\footnotetext{
${ }^{1}$ The organizational decision making has its roots in the seminal contributions of renowned mavens such as Simon, Cyert and March; for a comprehensive discussion of these issues, see [13].
} 


\section{Building the Foundation of a Cross-Policy Decision Support Framework}

In order to conceptualize a Decision Support Component (DSC) for the PADGETS platform we started from a set of key underlying assumptions regarding design principles as well as constraints we had to endure.

1. The design should be centered on the policy maker's perspective, focusing on the manifold needs of daily policy making.

2. The DSC as a whole has to be aligned to project mission and orientation: in particular the leitmotif to adhere to is the exploitation of many Social Media at the same time in a systematic and centrally managed manner.

3. Considering the economics of the project, reaching internal economies of scope represents for sure a desirable outcome. Thus, the effort has to be geared towards preventing the creation of non-communicating silos and towards avoiding the development "from scratch" of ad-hoc models for each specific pilot or locus of implementation.

4. In conceiving the application logic underpinning data elaboration, the novelty brought by PADGETS approach no longer considers individuals as isolated units of analysis but leverages their social connections and the context in which they are immersed as a potentially useful policy tool. By isolating particular behavior of specific groups, the policy maker may take advantage of an additional "weapon": by targeting more connected or more charismatic individuals s/he is likely to obtain better and faster results than by implementing a generic policy not taking into account the role individuals play in their social network.

5. Some potential threats pertain to the vast fields with which policy makers have to deal, such as the cognitive problem of synthesizing the distributed knowledge collected from stakeholders in many different environments and the intrinsic dynamics of public opinion. In light of such inescapable difficulties, it becomes paramount to keep moderate the cognitive effort required to policy makers while let the "machines" do most of the cumbersome work.

Keeping in mind afore-said cornerstones, we developed the architecture of a service that aims at informing the policy maker's decision process (i.e., a decision support tool) by effectively using the knowledge collected through the engagement with a plethora of stakeholders ${ }^{2}$ in Web 2.0 Social Media, i.e., virtual spaces which nowadays may ideally symbolize modern agorae.

Taking into consideration the rich variety of policy fields, we decided to develop a decision support tool capable to be as much as possible "generic" and "horizontal", meaning that it should be easily and effectively employed for any kind of public policy. This was done, among other reasons, to enhance the appeal of the Decision

\footnotetext{
${ }^{2}$ We prefer the generic term "stakeholders" to "citizens" because we think that citizens are only the largest kind of stakeholders interested in interacting with policy makers, and that institutions, which cannot be reduced to their single individuals, can be interested too in the innovative ways of participatory policy making introduced by the project. Hence, actors such as, for instance, producers' and consumers' associations, political parties, trade unions, corporations and charities, could be encompassed under the label "stakeholders".
} 
Support System in terms of commercialization, i.e., in order to be turned into a marketable product. As a matter of fact, the possibility to reach a wider pool of potential institutional adopters allows to benefit from economies of scope and scale, that contribute to lower the unit cost of service provision.

Moreover, considering the issue of synthesizing the widespread information collected through many different Web 2.0 participatory tools provided by the project, we started by interacting with local policy makers in order to identify the support they expect from such kind of a tool. Prominent desiderata coming from the "requirement phase" regard the potentiality of collecting through a unique tool various information stemming from dissimilar interaction patterns which are peculiar of different stages along the public policy lifecycle. In particular, policy makers would like to receive answers to the following five "archetypal" questions which are relevant during each phase of a public policy lifecycle ${ }^{3}$ (agenda setting, policy analysis, formulation, implementation, monitoring):

1. Are stakeholders aware of the public policy?

2. Are stakeholders interested in the public policy?

3. What stakeholders think about the specific public policy solution that the policy maker has proposed? To what extent they accept it?

4. Which are the barriers to policy awareness and interest, and which are the barriers to changes in public opinion about the policy?

5. Which suggestions are coming from stakeholders?

To say it with other words, the first question investigates if stakeholders know that the policy under examination exists; the second question regards to what extent they are inclined to reason and debate about the policy theme. The third point, for its part, is centered on stakeholders' judgment about the policy (e.g., acceptance, rejection, neutrality, indifference). The forth question strives for hints about barriers hampering the policy deployment as well as obstacles hindering the diffusion of the policy message. Finally, the fifth question has its root in the concept of "crowd wisdom", since it tries to collect insightful contributions coming from the collective opinion in an attempt to reap the benefits stemming from bottom-up knowledge percolation.

The identified relevant questions allowed us to design a support tool capable of taking advantage of the fruitful synergy among different methodologies and techniques. In order to devise responses to the first three questions, the most relevant information concerning the policy proposal collected from stakeholders are framed along three basic dimensions (awareness, interest, acceptance); on the other hand, in order to answer questions 4 and 5, stakeholders' suggestions are analyzed through text mining (which is performed on unstructured texts) and opinion analysis (which relies on structured interactions occurring through polls, questionnaires et similia).

We conceived the awareness-interest-acceptance framework by taking into account concrete needs of public policy makers and, at the same time, drawing from preeminent theoretical frameworks developed in the disciplines of innovation studies and political science. According to innovation research of Rogers [14], the diffusion of an innovation occurs, in fact, through a five-step process, which is a type of decision making: awareness, interest, evaluation, trial, and adoption. Furthermore,

\footnotetext{
${ }^{3}$ Afore-mentioned phases of the policy making cycle are defined by OECD in [15].
} 
OECD [16] identifies three stages of on-line engagement: information (for increasing stakeholders' awareness), consultation (providing opinions about the policy) and active engagement. In addition, the concept of policy acceptance is well-recognized in political science as it allows to understand the coherence between the proposed public action and the systems of values present in the society, a necessary precondition for a successful implementation of the policy; considering the literary landscape as well as down-to-earth policy initiatives, the concept of acceptance may be seen from a normative point of view or from innovation point of view ${ }^{4}$.

\section{The Architecture of the PADGETS Decision Support Component}

The PADGETS Decision Support Component is the analytic engine processing and analyzing the results of the PADGETS Campaign ${ }^{5}$ in order to extract useful information for the policy maker. To say it in a nutshell, it is the software component which prepares the information for supporting policy makers.

The DSC relies on information coming from the policy maker, from Social Media Platforms ${ }^{6}$, and from the Padget ${ }^{7}$, and consists of two main modules: the PADGETS Analytics and the PADGETS Simulation Model. Whilst the Analytics module aims at grouping and synthesizing raw information and at solving possible problems of statistical nature in collected data, the Simulation Model aspires to forecast future scenarios of opinion change.

In the following paragraphs we sketch the basic working mechanisms of the DSC, concluding with a discussion on the effectiveness of the proposed design both through the technical lens and the value-related lens.

\subsection{Inputs}

The inputs of the DSC model come from three sources, i.e., Social Media, Padgets and policy makers.

Data coming from Social Media (retrieved by public APIs) and from the Padgets may be unstructured (i.e., open text content) or, otherwise, structured (i.e., users' actions and selections). Unstructured data flow into text mining activities while structured data, for their part, constitute the inputs of quantitative analysis taking place in both PADGETS Analytics and PADGETS Simulation Model.

${ }^{4}$ For an example of EU funded research project on policy acceptance see [17].

${ }^{5}$ In the project jargon, a PADGETS Campaign entails a set of activities covering creation, distribution, interaction, monitoring and termination of one or more policy messages oriented towards a specific goal and related to the same theme.

${ }^{6}$ Major Social Media Platforms covered by the scope of work are: Facebook, LinkedIn, Twitter, Blogger, Digg, Scribd, YouTube, Picasa, Flickr. Currently the PADGETS consortium is examining the inclusion of the upcoming Google Plus in the Social Media panoply under investigation.

${ }^{7}$ In line with the project dictionary, a Padget is a resource (application or content), typically instantiating within a variety of Social Media Platforms, which provides interactivity with stakeholders through an ensemble of native and "augmented" social functionalities. 
The inputs database contains two broad categories of information in terms of data organization:

1. policy maker's data referring both to the target stakeholders' group (sociodemographic data) and to Campaign attributes (e.g., date of launch, timeframe, question structure);

2. data stemming from social engagement, collected at the finest granularity (i.e., individual users' data with recourse to appropriate techniques of anonymization, in compliance with data protection legal frameworks) and structured according to the two dimensions of user and time (i.e., the user who acted and the time of action).

\subsection{Output Indicators}

DSC outputs are developed along three different concepts in accordance with section 3: awareness, interest and acceptance.

From a Padget end user's perspective, each concept is a set containing the following ones, but not vice versa. Thus, a user interested in the policy must be also aware of the policy, but the opposite might not hold (i.e., an aware user can be not interested in the policy).

The distinction between the concepts is that acceptance concerns polarized judgments (i.e., positive and negative) collected by means of the Padget, interest regards all data generated by a proactive behavior by users in Social Media, and finally awareness is a matter of an only passive reception of the policy message in Social Media (i.e., without further spreading or commenting the Padget announcement owing to a lack of interest).

The typologies of outputs that it is possible to compute are three. Firstly, it is possible to draw the distribution of data over the main categories of stakeholders identifiable according to socio-demographic variables. Secondly, it is possible to project the data into the actual world. Lastly, estimates on how policy awarenessinterest-acceptance will change in the near future can be computed through algorithms included in the Simulation Model.

\subsection{Modeling and Simulation}

Keeping a helicopter view, the "big picture" of the PADGETS Decision Support Component structure (Fig. 1) displays how the two DSC modules transform the structured inputs coming from different sources in outputs useful for satiating policy makers' appetite, while an external module carries out text mining functionalities in order to determine stakeholders' suggestions. The figure, moreover, underlines that only the relevant information is presented to policy makers among the many we identified above.

Passing to the description of how the components work, actual distributions of awareness-interest-acceptance are obtained by a sort of mere data aggregation that simply groups raw Social Media and Padget data according to socio-demographic variables. 


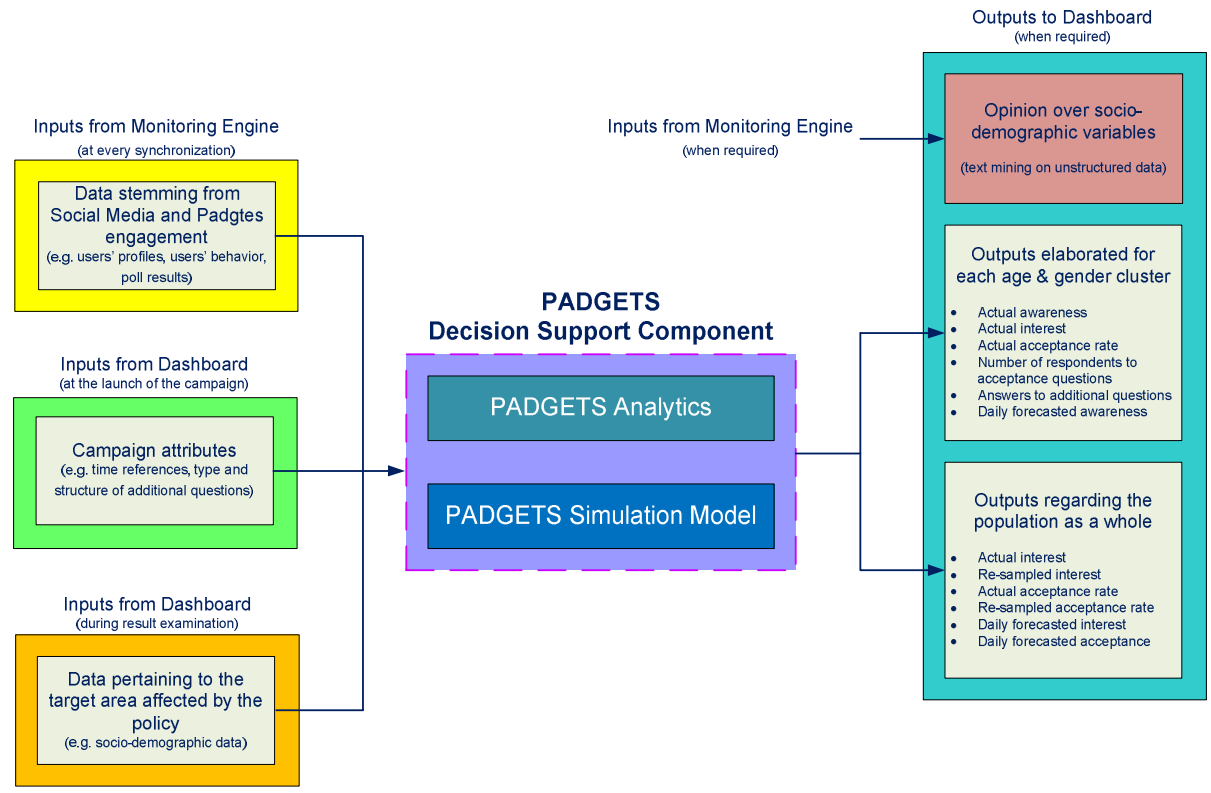

Fig. 1. The structure of the PADGETS Decision Support Component

In addition, in order to increase the real world significance of obtained results the re-sampling of raw data is computed: moving in this direction represents an attempt to remedy to possible underrepresentation of specific groups of stakeholders in the Social Media realm. To exemplify, elderly generations are likely to show lower penetration rates in Social Media: the resampling activity is thus aimed at reducing this bias (and several others) in the estimation of current and future awareness, interest and acceptance rates.

Finally, awareness-interest-acceptance future scenarios are the most complex results, representing the flagship of the DSC. They start from the re-sampling of raw data, on which a procedure computes trends evolution along the dimensions of policy indicators and in light of socio-demographic variables: for this purpose, a System Dynamics simulation is run in order to estimate the possible future outcomes and their probability of occurrence. Being the Simulation Model a System Dynamics one, there is the need for two main kinds of elements ${ }^{8}$, stocks and flows. Thus the Simulation Model is based on the identification of relevant stocks according to sociodemographic variables and flows depending on the above-mentioned trends of evolution. In particular, the simulation is focused on how distinct socio-demographic clusters of stakeholders will change their level of awareness-interest-acceptance in the near future in light of intertwined social connections and resulting "viral" contagious phenomena; the rationale underlying the model entails that clusters are not

${ }^{8}$ For a complete introduction to System Dynamics, to its concepts and terminology, please refer to [18]. 
independent, therefore several feedback loops and cascade effects can be at work testifying a blurred overlap of endogenous evolution and external influences.

Under a procedural point of view, the Simulation Model operates as follows.

1. The System Dynamics model is built-up automatically in the background in accordance with policy maker's input; by doing this, significant results are achieved in hiding the inherent complexity characterizing the Simulation Model from policy maker's eyes.

2. A calibration procedure is performed through a regression which computes coefficients and parameters of the model, estimating both mutual interrelations and endogenous growth in view of the evolution registered hitherto.

3. Given the stochastic nature of the simulation, the heterogeneity of collected data and the uncertainty affecting some parameters, the simulation will be run to explore all the possible outcomes of variations in parameters (including the random seed for stochastic processes). As a result, confidence intervals are computed with a certain level of confidence for each forecasted policy indicator: by moving in this direction, it is possible to shift the perspective from deterministic to probabilistic.

4. Once the simulation routines have run "behind the scene", fresh and customized results are passed to the front-end and presented to the policy maker in a compelling way through a fully-fledged web-based visualization engine.

\subsection{Effectiveness of the Solution}

An overall glance on the DSC shows that elaborated outputs directly answer the first three policy makers' questions out of the five we want to address (section 3): presenting to policy makers detailed data about the three concepts of awareness, interest and acceptance, they will obtain extensive answers to their recurrent questions and they will also get a "dynamic" idea about future trends in society towards the policy on focus.

The remaining two policy makers' questions we plan to answer pertain to perceived barriers and suggestions coming from stakeholders. In this case the answers to policy makers are provided by the DSC as a whole, that is to say by the results coming from the Analytics and the Simulation Model, on which we focused in this paper, and by analytic activities on unstructured data such as text mining.

Delving into this topic, the first three policy questions we considered as aims of the DSC are directly answered by singular groups of indicators provided by the DSC. For instance, if the policy maker is interested in evaluating the policy awareness in the population, the DSC provides a set of indicators related to awareness which describe the contemporary level of that phenomenon accompanied by its near future trend. On the other side, the two remaining questions concerning emerging barriers and public suggestions are per se unforeseeable in their structure and content, and it thus becomes impossible to identify ex ante a "rigid" structure to organize such information. For this purpose opinion mining [19] methods will be exploited. In this field, the effort is geared towards extracting opinions from unstructured humanauthored texts (posts, comments et similia) having recourse to techniques such as feature-based sentiment analysis, topic identification and sentiment classification. Semantic analyses in this vein provide an insightful glimpse on "what people think" capable to conspicuously reinforce the governmental policy intelligence. 
For the actual implementation "on the field" of the DSC architecture, we chose to rely on autonomous and platform independent software classes with data interfaces for communicating inputs and outputs with other building blocks belonging to the platform. Our choice has been to code this software entirely in Java (avoiding recourse to external libraries or tools for System Dynamics modeling) in order to guarantee platform independence, eventual Web distribution and for relying on well-established libraries devoted to required activities of data management and regression.

In conclusion, a pivotal aspect not to be overlooked is the compliance of the DSC with policy regulation and data protection legislation. In particular, all front-end operations required for data collection are performed after properly informing the user on subsequent processing activities in a precise and descriptive manner. PADGETS employs W3C's P3P [20], consequently for each PADGETS Campaign a P3P-compliant privacy policy is provided to inform the user about data elaboration within PADGETS in order to let him/her make a well-informed decision. Once obtained the positive user's consent, PADGETS consortium operates without storing users' IDs in a re-usable way. Finally, during the entire project lifetime there is no transfer of personal data to third parties: in fact, gathered data are stored on servers of one of the consortium partners inside the EU region and are owned by the consortium.

\subsection{From Code Implementation to Value Creation}

Since the "North Star" that guided our action is being markedly "value-driven" rather than being "tech-driven", in our opinion it may sound wise to conclude the paper by coming back to the policy maker's angle in order to pinpoint how the tool previously described is able to "make sense of data", smoothing the way for a better informed policy decision.

Summarizing, from a policy maker's perspective the value proposition of the decision support tool we designed may be recapitulated as follows:

1. A methodological contribution related to information classification, since the tool provides a well-grounded conceptual framework aimed to classify and aggregate data stemming from social engagement in light of an increasing level of stakeholders' involvement (awareness, interest, acceptance).

2. A reduction of information complexity, given by a set of peculiar traits (e.g., data aggregation along multiple dimensions, cross-platform data analysis, data projection into the real world, simulation of phenomena evolution in the near future) leading to a well-framed synthesis of unstructured (and sometimes inadvertent) society's input which could be used in order to forecast possible impacts of policies in light of surfacing vox populi.

3. A support to emerging Governance models, since it enables new ways for collecting, organizing and delivering information at different authority levels, opening-up on-going Governance models by letting a wider audience to contribute to the political debate.

\section{Conclusions and Limitations}

In this article we presented the preliminary results produced during the first year of activity of the PADGETS research project. In particular, the discussion focused on the 
policy analysis framework underpinning the DSC of the PADGETS platform. Such analytical framework was generated through a process of interdisciplinary integration (mainly drawing form the diffusion of innovation and political science literature) as well as through a process of end users needs elicitation.

The intent behind the development of the analytical framework was to provide a first contribution towards the creation of a Decision Support System that could help policy makers in facing a number of relevant questions often arising through the policy cycle. This was done by introducing an innovation bringing together Social Networks and System Dynamics simulation. To date, in fact, the use of ICT tools for decision support has traditionally been a "closed door" activity usually carried out with static external inputs in the form of codified or unstructured data coming from different sources (e.g., statistical offices). Such approach presents a number of important limitations: evident examples are the lack of a direct connection with the recent external reality on which the policy decision has to impact and the inherent delay present in the policy response due to the lead time necessary to collect and process the relevant data required for the analysis. To illustrate with a metaphor, such process could be compared to driving a car by only looking at the rear view mirror (a partial, indirect and delayed input) rather than through the windscreen. The innovation brought by PADGETS consists in opening up the decision support process by integrating it with the activities carried out over Social Media Platforms. This allows to establish a direct link between the decision process and the external world as well as to reason on fresh and relevant information. This, once the necessary organizational processes are in place, should contribute to produce a much more responsive and effective style of decision making in Government. Going back to our metaphor, the innovation introduced by the Decisions Support Component of PADGETS aims at allowing decision makers to drive looking through the windscreen supported by an intelligent navigation system able to anticipate some of the obstacles lying ahead (i.e., the predictive functionalities of the simulation module).

Finally, it is important to discuss also some of the limitations that characterize the solution presented, as they may represent an interesting starting point for future research. The resampling activity used for the generalization of the results in terms of interest and acceptance, for example, contributes to decrease some of the biases inherent in Social Media usage (e.g., age distribution) but it is far from producing a statistically significant representation of society. In addition, the implementation of a meaningful cross-platform tracking systems still presents a number of challenges having to do with identity management. Along these lines, potential criticalities could derive also in case of scarcity of personal information regarding end users due to heterogeneous policies adopted by Social Media Platforms as well as end users' privacy settings: even though the robustness of the Simulation Model has been repeatedly tested in "borderline" use-cases, the paucity of a Minimum Set of Data (basically users' age and gender, that represent key variables on which the clustering procedure is based) may reduce the representativeness of final results and, consequently, could lower the quality of elaborated reports.

Concluding, although far from being error free, it is our firm belief that the framework presented constitutes a significant step ahead in helping policy makers in dealing with the challenges arising from the complexity that more and more may be found in modern societies. 


\section{References}

1. Bauman, Z.: Liquid Modernity. Polity Press, Cambridge (2000)

2. PADGETS Project, http: / / www . padgets . eu

3. Sterman, J.D.: Learning in and about complex systems. System Dynamics Review 10(2-3), 291-330 (1994)

4. Courtney, J.F.: Decision making and knowledge management in inquiring organizations: toward a new decision-making paradigm for DSS. Decision Support Systems 31(1), 17-38 (2001)

5. Beers, P.J., Boshuizen, H.P.A.E., Kirschner, P.A., Van den Bossche, P.: Decision-support and Complexity in Decision Making. In: 5th Junior Researchers of EARLI (JURE) Conference, Amsterdam, The Netherlands (2002)

6. Nonaka, I.: A dynamic theory of organizational knowledge creation. Organization Science 5(1), 14-37 (1994)

7. Brabham, D.C.: Crowdsourcing as a model for problem solving: An introduction and cases. Convergence: The International Journal of Research into New Media Technologies 14(1), 75-90 (2008)

8. Benčina, J.: Web-based Decision Support System for the Public Sector Comprising Linguistic Variables. Informatica 31, 311-323 (2007)

9. Kamel, S.: Decision Support Systems and Strategic Public Sector Decision Making in Egypt. Paper No. 3, Working Paper Series in Information Systems for Public Sector Management, Institute for Development Policy and Management, Manchester, UK (1998)

10. Osimo, D.: Web 2.0 in Government: Why and How. In: JRC Scientific and Technical Reports, European Commission, Joint Research Centre, Institute for Prospective Technological Studies (2008)

11. Charalabidis, Y., Gionis, G., Ferro, E., Loukis, E.: Towards a Systematic Exploitation of Web 2.0 and Simulation Modeling Tools in Public Policy Process. In: Tambouris, E., Macintosh, A., Glassey, O. (eds.) ePart 2010. LNCS, vol. 6229, pp. 1-12. Springer, Heidelberg (2010)

12. Lévy, P.: Collective intelligence: Mankind's emerging world in cyberspace. Perseus Books, Cambridge (1997)

13. Shim, J.P., Warkentin, M., Courtney, J.F., Power, D.J., Sharda, R., Carlsson, C.: Past, present, and future of decision support technology. Decision Support Systems 33(2), 111126 (2002)

14. Rogers, E.M.: Diffusion of Innovations. Free Press, New York (1983)

15. OECD: Policy Brief: Engaging Citizens Online for Better Policy-Making. OECD Observer (2003)

16. OECD: Citizens as Partners: Information, Consultation and Public Participation in PolicyMaking. OECD Publishing (2001)

17. European Commission: Population Policy Acceptance Study - The Viewpoint of Citizens and Policy Actors Regarding the Management of Population Related Change. Final report, EU Research on Social Sciences and Humanities, Brussels (2006)

18. Forrester, J.W.: Industrial Dynamics. The MIT Press, Cambridge (1961)

19. Pang, B., Lee, L.: Opinion mining and sentiment analysis. Foundations and Trends in Information Retrieval 2(1-2), 1-135 (2008)

20. W3C's Platform for Privacy Preferences (P3P), http: / / www . w3 . org / P3P 